\title{
An Improved Belief Entropy and Its Application in Decision-Making
}

\author{
Deyun Zhou, Yongchuan Tang, and Wen Jiang \\ School of Electronics and Information, Northwestern Polytechnical University, Xian, Shaanxi 710072, China \\ Correspondence should be addressed to Yongchuan Tang; tangyongchuan@mail.nwpu.edu.cn \\ and Wen Jiang; jiangwen@nwpu.edu.cn
}

Received 22 December 2016; Accepted 23 January 2017; Published 16 March 2017

Academic Editor: Jurgita Antucheviciene

Copyright (C) 2017 Deyun Zhou et al. This is an open access article distributed under the Creative Commons Attribution License, which permits unrestricted use, distribution, and reproduction in any medium, provided the original work is properly cited.

Uncertainty measure in data fusion applications is a hot topic; quite a few methods have been proposed to measure the degree of uncertainty in Dempster-Shafer framework. However, the existing methods pay little attention to the scale of the frame of discernment (FOD), which means a loss of information. Due to this reason, the existing methods cannot measure the difference of uncertain degree among different FODs. In this paper, an improved belief entropy is proposed in Dempster-Shafer framework. The proposed belief entropy takes into consideration more available information in the body of evidence (BOE), including the uncertain information modeled by the mass function, the cardinality of the proposition, and the scale of the FOD. The improved belief entropy is a new method for uncertainty measure in Dempster-Shafer framework. Based on the new belief entropy, a decisionmaking approach is designed. The validity of the new belief entropy is verified according to some numerical examples and the proposed decision-making approach.

\section{Introduction}

Decision-making in the uncertain environment is common in real world applications, such as civil engineering [1-3], supply chain management $[4,5]$, risk analysis $[6,7]$, medical service $[8,9]$, and so on $[10-12]$. In order to reach the goal of maximum comprehensive benefits, many methods have been developed for decision-making based on probability theory [13, 14], fuzzy set theory [15-18], Dempster-Shafer evidence theory [19-22], rough set theory [23-25], and so on [26-29].

Dempster-Shafer evidence theory $[19,20]$ is effective in uncertain information processing. It provides the frame of discernment (FOD) and the basic probability assignment (BPA) for information modeling, as well as the Dempster's rule of combination for data fusion. Dempster-Shafer evidence theory has been extensively studied in many fields such as pattern recognition [30-34], fault diagnosis [35-38], multiple attribute decision-making [39-41], risk analysis [22, $35,42,43]$, controller design $[44,45]$, and so on [4648]. Some open issues in Dempster-Shafer evidence theory are still needed for further study, including the conflicting management [49-52], the independence of different evidences [53-55], the methods to generate BPAs [56-58], and the incompleteness of the FOD [59-61]. One way to address these open issues is to quantify the uncertain degree of uncertain information before further information processing.

In the probabilistic framework, Shannon entropy [62] is a well-known theory for uncertainty measure; it has attracted much attention in real applications [63-65]. But Shannon entropy cannot be used directly in the framework of Dempster-Shafer evidence theory, because a mass function in evidence theory is a generalized probability assigned on the power set of the FOD. In order to overcome this limitation, in Dempster-Shafer framework, many methods have been proposed to measure the uncertain degree of the evidence, such as Hohle's confusion measure [66], Yager's dissonance measure [67], Dubois \& Prade's weighted Hartley entropy [68], Klir \& Ramer's discord measure [69], Klir \& Parviz's strife measure [70], George \& Pal's total conflict measure [71], and so on [72-74]. Some of these methods are derived from Shannon entropy [66-70]. But the aforementioned methods are somehow not that effective in some cases $[71,75]$. 
Lately, another uncertainty measure named Deng entropy [75] is proposed in Dempster-Shafer framework. Although Deng entropy has been successfully applied in some real applications [36-38, 80,81], it does not take into consideration the scale of the FOD, which means a loss of available information while doing information processing. To make matters worse, the information loss will lead to failure for uncertainty measure in some cases [71]. The same shortage also exists in the confusion measure [66], the dissonance measure [67], the weighted Hartley entropy [68], the discord measure [69], and the strife measure [70]. To address this issue, an improved belief entropy based on Deng entropy is proposed in this paper. The proposed belief entropy can improve the performance of Deng entropy by considering the scale of the FOD and the relative scale of a focal element with respect to FOD. What is more, the proposed method keeps all the merits of Deng entropy; thus it can degenerate to Shannon entropy in the sense of the probability consistency.

In order to verify the validity of the improved belief entropy, a decision-making approach in target identification is designed based on the new belief entropy. In the proposed method, the uncertain degree of the sensor data is measured by the new belief entropy; then the uncertain degree will be used as the relative weight of each sensor report modeled as the body of evidence (BOE); after that, the BPAs of the BOE will be modified by the weight value; finally, the decision-making is based on the fusion results of applying Dempster's rule of combination to the modified BPAs.

The rest of this paper is organized as follows. In Section 2, the preliminaries on Dempster-Shafer evidence theory, Shannon entropy, and some uncertainty measures in DempsterShafer framework are briefly introduced. In Section 3, the improved belief entropy is proposed; some behaviors of the proposed belief entropy are discussed with the numerical examples. In Section 4, an uncertainty measure-based decision-making approach is proposed to show the efficiency of the new belief entropy. The conclusions are given in Section 5 .

\section{Preliminaries}

In this section, some preliminaries are briefly introduced, including Dempster-Shafer evidence theory, Shannon entropy, and some typical uncertainty measures in DempsterShafer framework.

2.1. Dempster-Shafer Evidence Theory. Let $\Omega=\left\{\theta_{1}, \theta_{2}, \ldots\right.$, $\left.\theta_{i}, \ldots, \theta_{N}\right\}$ be a finite nonempty set of mutually exclusive and exhaustive events, and $\Omega$ is called the frame of discernment (FOD). The power set of $\Omega$, denoted as $2^{\Omega}$, is composed of $2^{N}$ elements denoted as follows:

$$
\begin{aligned}
& 2^{\Omega}=\left\{\emptyset,\left\{\theta_{1}\right\},\left\{\theta_{2}\right\}, \ldots,\left\{\theta_{N}\right\},\left\{\theta_{1}, \theta_{2}\right\}, \ldots,\left\{\theta_{1}, \theta_{2}, \ldots, \theta_{i}\right\},\right. \\
& \ldots, \Omega\} .
\end{aligned}
$$

A mass function $m$ is defined as a mapping from the power set $2^{\Omega}$ to the interval $[0,1]$, which satisfies the following conditions $[19,20]$ :

$$
\begin{aligned}
m(\emptyset) & =0, \\
\sum_{A \in \Omega} m(A) & =1 .
\end{aligned}
$$

If $m(A)>0$, then $A$ is called a focal element, and the mass function $m(A)$ represents how strongly the evidence supports proposition $A$.

A body of evidence (BOE), also known as a basic probability assignment (BPA) or basic belief assignment (BBA), is represented by the focal sets and their associated mass value:

$$
(\Re, m)=\left\{\langle A, m(A)\rangle: A \in 2^{\Omega}, m(A)>0\right\},
$$

where $\Re$ is a subset of the power set $2^{\Omega}$ and each $A \in \mathfrak{R}$ has an associated nonzero mass value $m(A)$.

A BPA $m$ can also be represented by its associate belief function Bel and plausibility function $\mathrm{Pl}$, respectively, defined as follows:

$$
\begin{aligned}
\operatorname{Bel}(A) & =\sum_{\phi \neq B \subseteq A} m(B), \\
\operatorname{Pl}(A) & =\sum_{B \cap A \neq \phi} m(B) .
\end{aligned}
$$

In Dempster-Shafer evidence theory, two independent mass functions, denoted as $m_{1}$ and $m_{2}$, can be combined with Dempster's rule of combination defined as follows $[19,20]$ :

$$
m(A)=\left(m_{1} \oplus m_{2}\right)(A)=\frac{1}{1-k} \sum_{B \cap C=A} m_{1}(B) m_{2}(C),
$$

where $k$ is a normalization constant representing the degree of conflict between $m_{1}$ and $m_{2}$ and $k$ is defined as follows $[19,20]$ :

$$
k=\sum_{B \cap C=\emptyset} m_{1}(B) m_{2}(C) .
$$

2.2. Shannon Entropy. As an uncertainty measure of information volume in a system or process, Shannon entropy plays a central role in information theory. Shannon entropy indicates that the information volume of each piece of information is directly connected to its uncertainty degree.

Shannon entropy, as the information entropy, is defined as follows [62]:

$$
H=-\sum_{i=1}^{N} p_{i} \log _{b} p_{i}
$$

where $N$ is the number of basic states, $p_{i}$ is the probability of state $i$ and $p_{i}$ satisfies $\sum_{i=1}^{N} p_{i}=1$, and $b$ is the basis of the logarithm which accounts for the scaling of $H$. Although $b$ is arbitrary, $b$ is usually chosen to be 2 , and the unit of information entropy is bit. If $b$ is the natural base, then the 
TABLE 1: Uncertainty measures in Dempster-Shafer framework.

\begin{tabular}{ll}
\hline Uncertainty measure & Definition \\
\hline Hohle's confusion measure [66] & $C_{\mathrm{H}}(m)=-\sum_{A \subseteq X} m(A) \log _{2} \operatorname{Bel}(A)$ \\
Yager's dissonance measure [67] & $E_{\mathrm{Y}}(m)=-\sum_{A \subseteq X} m(A) \log _{2} \mathrm{Pl}(A)$ \\
Dubois \& Prade's weighted Hartley entropy [68] & $E_{\mathrm{DP}}(m)=\sum_{A \subseteq X} m(A) \log _{2}|A|$ \\
Klir \& Ramer's discord measure [69] & $D_{\mathrm{KR}}(m)=-\sum_{A \subseteq X} m(A) \log _{2} \sum_{B \subseteq X} m(B) \frac{|A \cap B|}{|B|}$ \\
Klir \& Parviz's strife measure [70] & $S_{\mathrm{KP}}(m)=-\sum_{A \subseteq X} m(A) \log _{2} \sum_{B \subseteq X} m(B) \frac{|A \cap B|}{|A|}$ \\
George \& Pal's total conflict measure [71] & $T C_{\mathrm{GP}}(m)=\sum_{A \subseteq X} m(A) \sum_{B \subseteq X} m(B)\left(1-\frac{|A \cap B|}{|A \cup B|}\right)$ \\
\hline
\end{tabular}

unit of information entropy will be Nat. Mathematically, as a scaling factor, different basis of the logarithm is convertible.

2.3. Uncertainty Measures in Dempster-Shafer Framework. In Dempster-Shafer framework, some uncertainty measures for the $\mathrm{BOE}$ are presented, as is shown in Table 1 , where $X$ is the FOD, $A$ and $B$ are focal elements of a mass function, and $|A|$ denotes the cardinality of $A$.

Lately, another belief entropy, named Deng entropy, is presented to measure the uncertainty in the BOE. Deng entropy, denoted as $E_{d}$, is defined as follows [75]:

$$
E_{d}(m)=-\sum_{A \subseteq X} m(A) \log _{2} \frac{m(A)}{2^{|A|}-1} .
$$

\section{The Improved Belief Entropy}

3.1. Problem Description. In Dempster-Shafer framework, the uncertain information modeled in the BOE includes the mass function and the FOD. However, the existing uncertainty measures only focus on the mass function [66, 67] or at most take into consideration the cardinality of each proposition $[68-71,75]$. In other words, the scale of the FOD is totally ignored. Without taking full advantage of available information in the BOE, the existing uncertainty measures cannot effectively quantify the difference among different BOEs if the same mass value is assigned on different FOD. A simple example of the limitation of Deng entropy is shown in Example 1.

Example 1. Consider a target identification problem; assume that two reliable sensors report the detection results independently. The results are represented by BOEs shown as follows:

$$
\begin{aligned}
m_{1}: m_{1}(\{a, b\}) & =0.4, \\
m_{1}(\{c, d\}) & =0.6, \\
m_{2}: m_{2}(\{a, c\}) & =0.4, \\
m_{2}(\{b, c\}) & =0.6 .
\end{aligned}
$$

Recalling (8), the uncertainty measure with Deng entropy is shown as follows:

$$
\begin{aligned}
E_{d}\left(m_{1}\right) & =-\sum_{A \subseteq X} m_{1}(A) \log _{2} \frac{m_{1}(A)}{2^{|A|}-1} \\
& =-0.4 \log _{2} \frac{0.4}{2^{2}-1}-0.6 \log _{2} \frac{0.6}{2^{2}-1} \\
& =2.5559, \\
E_{d}\left(m_{2}\right) & =-\sum_{A \subseteq X} m_{2}(A) \log _{2} \frac{m_{2}(A)}{2^{|A|}-1} \\
& =-0.4 \log _{2} \frac{0.4}{2^{2}-1}-0.6 \log _{2} \frac{0.6}{2^{2}-1} \\
& =2.5559 .
\end{aligned}
$$

The limitation of Deng entropy also exists in Dubois \& Prade's weighted Hartley entropy [68], and the uncertainty measure with the weighted Hartley entropy is shown as follows:

$$
\begin{aligned}
E_{\mathrm{DP}}\left(m_{1}\right) & =\sum_{A \subseteq X} m_{1}(A) \log _{2}|A| \\
& =0.4 \log _{2} 2+0.6 \log _{2} 2=1, \\
E_{\mathrm{DP}}\left(m_{2}\right) & =\sum_{A \subseteq X} m_{2}(A) \log _{2}|A| \\
& =0.4 \log _{2} 2+0.6 \log _{2} 2=1 .
\end{aligned}
$$

The results calculated by Deng entropy and the weighted Hartley entropy are counterintuitive. Although the two BOEs have the same mass value, the FOD of the first BOE $m_{1}$ consists of four targets, denoted as $a, b, c$, and $d$, while the second $\mathrm{BOE} m_{2}$ has only three possible targets, denoted as $a, b$, and $c$. Intuitively, it is expected that $m_{2}$ has a less uncertainty than $m_{1}$. In other words, the belief entropy of $m_{1}$ should be bigger than that of $m_{2}$. Both Deng entropy and weighted Hartley entropy fail to quantify this difference. 
TABLE 2: Calculation results in Example 1.

\begin{tabular}{lccc}
\hline BOEs & Weight Hartley entropy [68] & Deng entropy [75] & Improved belief entropy \\
\hline$m_{1}$ & 1 & 2.5559 & 2.1952 \\
$m_{2}$ & 1 & 2.5559 & 2.0750 \\
\hline
\end{tabular}

To address this issue, an improved belief entropy is proposed.

3.2. The Proposed Belief Entropy. In Dempster-Shafer framework, the improved belief entropy is proposed as follows:

$$
E_{I d}(m)=-\sum_{A \subseteq X} m(A) \log _{2}\left(\frac{m(A)}{2^{|A|}-1} e^{(|A|-1) /|X|}\right)
$$

where $X$ is the FOD, $|A|$ denotes the cardinality of the focal element $A$, and $|X|$ is the number of element in the FOD. Compared with some other uncertainty measures in [66-71, 75], the improved belief entropy addresses more information in a BOE. The uncertain information addressed by the new belief entropy includes the information represented by the mass function, the cardinality of each proposition, the scale of FOD (denoted as $|X|$ ), and the relative scale of a focal element with respect to the FOD (denoted as $((|A|-1) /|X|))$.

In detail, compared with the confusion measure [66] and the dissonance measure [67], the uncertain information modeled by the cardinality of each proposition and the scale of FOD now can be handled properly. Compared with the weighted Hartley entropy [68], the discord measure [69], the strife measure [70], the total conflict measure [71] and Deng entropy [75], the scale of FOD, and the relative scale of a focal element with respect to the FOD now can be processed effectively. Above all, by involving the cardinality of each proposition and the scale of FOD in the proposed belief entropy, the new uncertainty measure can now effectively quantify the difference among different BOEs even if the same mass value is assigned on different FODs. What is more, more information of the BOE is addressed in information processing which means less information loss.

With the new belief entropy, recall the issue in Example 1; the improved belief entropy for these two BOEs is calculated as follows:

$$
\begin{aligned}
E_{I d}\left(m_{1}\right)= & -\sum_{A \subseteq X} m_{1}(A) \log _{2}\left(\frac{m_{1}(A)}{2^{|A|}-1} e^{(|A|-1) /|X|}\right) \\
= & -0.4 \log _{2}\left(\frac{0.4}{2^{2}-1} e^{(2-1) / 4}\right) \\
& -0.6 \log _{2}\left(\frac{0.6}{2^{2}-1} e^{(2-1) / 4}\right)=2.1952, \\
E_{I d}\left(m_{2}\right)= & -\sum_{A \subseteq X} m_{2}(A) \log _{2}\left(\frac{m_{2}(A)}{2^{|A|}-1} e^{(|A|-1) /|X|}\right)
\end{aligned}
$$

$$
\begin{aligned}
= & -0.4 \log _{2}\left(\frac{0.4}{2^{2}-1} e^{(2-1) / 3}\right) \\
& -0.6 \log _{2}\left(\frac{0.6}{2^{2}-1} e^{(2-1) / 3}\right)=2.0750 .
\end{aligned}
$$

It can be concluded that both the weighted Hartley entropy and Deng entropy cannot measure the different uncertain degree between these two BOEs, while the new belief entropy can effectively measure the difference by taking into consideration more available information of the BOE. According to Table 2, it is also safe to say that the first BOE $m_{1}$ has a higher uncertain degree with the new belief entropy; this is reasonable because the FOD of $m_{1}$ includes four possible targets which means a larger information volume than the second BOE $m_{2}$. The efficiency of the new belief entropy is not available in the weighted Hartley entropy and Deng entropy.

3.3. Behaviors of the Proposed Belief Entropy. In order to show the rationality and merit of the proposed belief entropy, some numerical examples are presented in this section. In Section 3.3.1, the compatibility of the new belief entropy with Shannon entropy is verified with some simple numerical examples. In Section 3.3.2, the superiority of the new belief entropy compared with some other uncertainty measures is presented.

\subsubsection{Compatibility with Shannon Entropy}

Example 2. Consider a target identification problem; if the target reported by the sensor is $a$ with one hundred percent belief, then the mass function can be denoted as $m(\{a\})=1$ in the frame of discernment $X=\{a\}$.

Shannon entropy $H$, Deng entropy $E_{d}$, and the improved belief entropy $E_{I d}$ are calculated, respectively, as follows:

$$
\begin{aligned}
H(m) & =-1 \times \log _{2} 1=0, \\
E_{d}(m) & =-1 \times \log _{2} \frac{1}{2^{1}-1}=0, \\
E_{I d}(m) & =-1 \times \log _{2}\left(\frac{1}{2^{1}-1} e^{(1-1) / 1}\right)=0 .
\end{aligned}
$$

It is obvious that the uncertainty degree for a certain event is zero. So the values of Shannon entropy, Deng entropy, and the improved belief entropy are all zero.

Example 3. Consider the mass functions $m(\{a\})=m(\{b\})=$ $m(\{c\})=m(\{d\})=0.25$ in the frame of discernment $X=$ $\{a, b, c, d\}$. 
TABLE 3: Improved belief entropy with a variable element number in $T$.

\begin{tabular}{lcc}
\hline Cases & Deng entropy & Improved belief entropy \\
\hline$T=\{1\}$ & 2.6623 & 2.5180 \\
$T=\{1,2\}$ & 3.9303 & 3.7090 \\
$T=\{1,2,3\}$ & 4.9082 & 4.6100 \\
$T=\{1, \ldots, 4\}$ & 5.7878 & 5.4127 \\
$T=\{1, \ldots, 5\}$ & 6.6256 & 6.1736 \\
$T=\{1, \ldots, 6\}$ & 7.4441 & 6.9151 \\
$T=\{1, \ldots, 7\}$ & 8.2532 & 7.6473 \\
$T=\{1, \ldots, 8\}$ & 9.0578 & 8.3749 \\
$T=\{1, \ldots, 9\}$ & 9.8600 & 9.1002 \\
$T=\{1, \ldots, 10\}$ & 10.6612 & 9.8244 \\
$T=\{1, \ldots, 11\}$ & 11.4617 & 10.5480 \\
$T=\{1, \ldots, 12\}$ & 12.2620 & 11.2714 \\
$T=\{1, \ldots, 13\}$ & 13.0622 & 11.9946 \\
$T=\{1, \ldots, 14\}$ & 13.8622 & 12.7177 \\
\hline
\end{tabular}

Shannon entropy $H$, Deng entropy $E_{d}$, and the improved belief entropy $E_{I d}$ are calculated, respectively, as follows:

$$
\begin{aligned}
H(m) & =\left(-0.25 \times \log _{2} 0.25\right) \times 4=2.0000, \\
E_{d}(m) & =\left(-0.25 \times \log _{2} \frac{0.25}{2^{1}-1}\right) \times 4=2.0000, \\
E_{I d}(m) & =\left(-0.25 \times \log _{2}\left(\frac{0.25}{2^{1}-1} e^{(1-1) / 4}\right)\right) \times 4 \\
& =2.0000 .
\end{aligned}
$$

According to Examples 2 and 3, if the mass value is only assigned on the single element, the result of the improved belief entropy is consistent with Shannon entropy and Deng entropy. The new belief entropy is compatible with Shannon entropy in the sense of the probability consistency, which indicates the effectiveness of the proposed belief entropy.

3.3.2. Efficiency in Uncertainty Measure. In order to test the efficiency and merit of the new belief entropy, recall the numerical example in [75] as follows.

Example 4. Consider the mass functions $m(\{6\})=0.05$, $m(\{3,4,5\})=0.05, m(T)=0.8$, and $m(X)=0.1$. The FOD $X=\{1,2, \ldots, 14,15\}$ is with fifteen elements denoted as Element $1, \ldots$, and Element 15 . The proposition $T$ consists of a variable subset with the number of element changing from one to fourteen, as is shown in Table 3.

Deng entropy $E_{d}$ and the improved belief entropy $E_{I d}$ are calculated, respectively, with the variable element number in the proposition $T$; the results are shown in Table 3 . Table 3 shows that the improved belief entropy is smaller than Deng entropy. This is reasonable, because more information in the $\mathrm{BOE}$ is taken into consideration within the improved

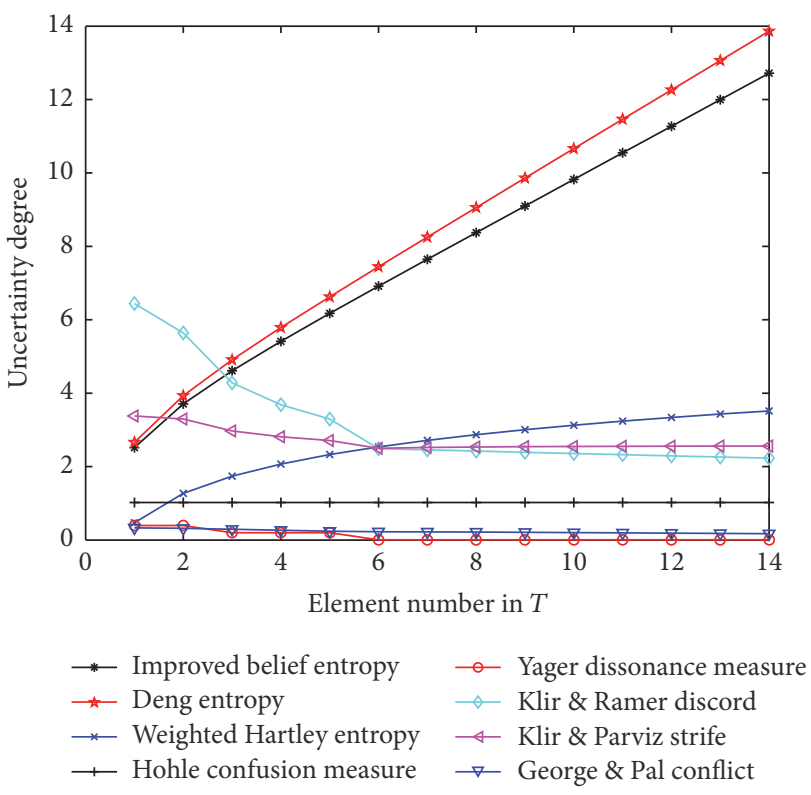

FIGURE 1: Comparison among different uncertainty measures.

belief entropy. By taking into consideration more available information, the uncertain degree measured by the new belief entropy decreases significantly compared to Deng entropy, which can be really helpful in information processing.

The uncertain degree in Example 4, measured by the other uncertainty measures in Table 1, is shown in Figure 1. The uncertain degree measured by Hohle's confusion measure never changes with the variation of the element number in proposition $T$; thus it cannot measure the variance of uncertain degree in this case. Similar to Hohle's confusion measure, Yager's dissonance measure has a limited capacity of uncertainty measure in this case. Both the confusion measure and the dissonance measure cannot measure the change in the proposition $T$. The uncertain degree measured by Klir \& Ramer's discord measure, Klir \& Parviz's strife measure, and George \& Pal's conflict measure is decreasing with the increasing of the element number in the proposition $T$; this is counterintuitive. Thus, the confusion measure, the dissonance measure, the discord measure, the strife measure, and the conflict measure cannot effectively measure the rising of the uncertain degree along with the increasing of the element number in the proposition $T$.

It seems that the uncertain degree measured by Dubois \& Prade's weighted Hartley entropy, Deng entropy, and the modified belief entropy is rising significantly with the increasing of the element number in proposition $T$. However, the weighted Hartley entropy and Deng entropy cannot distinguish the different uncertain degree among the BOEs with similar BPAs on different FODs, as is shown in Example 1. Thus, the improved belief entropy is the only available method for uncertainty measure in this case. More importantly, the proposed belief entropy takes advantage of more valuable information in the BOE, which ensures it to be more reasonable and effective for uncertainty measure in Dempster-Shafer framework. 


\section{An Uncertainty Measure-Based Decision-Making Approach}

In this section, a decision-making approach in sensor data fusion is presented. After uncertainty measure with the improved belief entropy, the modified BOEs are fused with Dempster's rule of combination. Finally, decision-making is based on the fused results.

4.1. Problem Description. In target recognition, sometimes, decision-making is based on reports from sensors. Consider the problem in [78]; the potential objects in the target recognition system are denoted as $a, b$, and $c$ in the FOD, denoted as $X=\{a, b, c\}$. Five sensors report on the possible object independently and successively; each report is represented as a BOE; these BOEs are denoted as $m_{1}, m_{2}, m_{3}, m_{4}$, and $m_{5}$, respectively.

$$
\begin{aligned}
m_{1}: m_{1}(\{a\}) & =0.41, \\
m_{1}(\{b\}) & =0.29, \\
m_{1}(\{c\}) & =0.30, \\
m_{1}(\{a, c\}) & =0.00, \\
m_{2}: m_{2}(\{a\}) & =0.00, \\
m_{2}(\{b\}) & =0.90, \\
m_{2}(\{c\}) & =0.10, \\
m_{2}(\{a, c\}) & =0.00, \\
m_{3}: m_{3}(\{a\}) & =0.58, \\
m_{3}(\{b\}) & =0.07, \\
m_{3}(\{c\}) & =0.00, \\
m_{3}(\{a, c\}) & =0.35, \\
m_{4}: m_{4}(\{a\}) & =0.55, \\
m_{5}: m_{5}(\{a\}) & =0.60, \\
m_{5}(\{b\}) & =0.10,10, \\
m_{5}(\{c\}) & =0.00, \\
m_{4}(\{c\}) & =0.00, \\
m_{5}(\{a, c\}) & =0.30,
\end{aligned}
$$

With the incoming data of sensors, which is the target? The target recognition system needs to make a decision based on the fusion results of the sensor data. Intuitively, $a$ will be the right target. In the second $\mathrm{BOE}$, the mass value assigned on target $a$ is zero and the mass value of target $b$ is 0.9 ; this is in high conflict with the other BOEs. According to [78, 79], the second BOE $m_{2}$ may come from a bad sensor and its report on the target is abnormal. $m_{2}$ will infer the correct fusion result, which may lead to a failure in decision-making. This case can be really a challenge for some data fusion methods that cannot handle conflicting evidences effectively $[49,50,52]$.

4.2. Uncertainty Measure with the New Belief Entropy. Before further addressing the BOEs reported by sensors, the quality of this information is quantified with the proposed belief entropy. Recalling (12), the improved belief entropy of each BOE is calculated as follows:

$$
\begin{aligned}
E_{I d}\left(m_{1}\right)= & -\sum_{A \subseteq X} m_{1}(A) \log _{2}\left(\frac{m_{1}(A)}{2^{|A|}-1} e^{(|A|-1) /|X|}\right) \\
= & -0.41 \log _{2}\left(\frac{0.41}{2^{1}-1} e^{(1-1) / 3}\right) \\
& -0.29 \log _{2}\left(\frac{0.29}{2^{1}-1} e^{(1-1) / 3}\right) \\
& -0.3 \log _{2}\left(\frac{0.3}{2^{1}-1} e^{(1-1) / 3}\right) \\
= & 0.5274+0.5179+0.5211=1.5664
\end{aligned}
$$

$$
\begin{aligned}
E_{I d}\left(m_{2}\right)= & -\sum_{A \subseteq X} m_{2}(A) \log _{2}\left(\frac{m_{2}(A)}{2^{|A|}-1} e^{(|A|-1) /|X|}\right) \\
= & -0.9 \log _{2}\left(\frac{0.9}{2^{1}-1} e^{(1-1) / 3}\right) \\
& -0.1 \log _{2}\left(\frac{0.1}{2^{1}-1} e^{(1-1) / 3}\right) \\
= & 0.1368+0.3322=0.4690
\end{aligned}
$$

$$
\begin{aligned}
E_{I d}\left(m_{3}\right)= & -\sum_{A \subseteq X} m_{3}(A) \log _{2}\left(\frac{m_{3}(A)}{2^{|A|}-1} e^{(|A|-1) /|X|}\right) \\
= & -0.58 \log _{2}\left(\frac{0.58}{2^{1}-1} e^{(1-1) / 3}\right) \\
& -0.07 \log _{2}\left(\frac{0.07}{2^{1}-1} e^{(1-1) / 3}\right) \\
& -0.35 \log _{2}\left(\frac{0.35}{2^{2}-1} e^{(2-1) / 3}\right) \\
= & 0.4558+0.2686+0.9165=1.6409
\end{aligned}
$$

$$
\begin{aligned}
E_{I d}\left(m_{4}\right)= & -\sum_{A \subseteq X} m_{4}(A) \log _{2}\left(\frac{m_{4}(A)}{2^{|A|}-1} e^{(|A|-1) /|X|}\right) \\
= & -0.55 \log _{2}\left(\frac{0.55}{2^{1}-1} e^{(1-1) / 3}\right) \\
& -0.1 \log _{2}\left(\frac{0.1}{2^{1}-1} e^{(1-1) / 3}\right) \\
& -0.35 \log _{2}\left(\frac{0.35}{2^{2}-1} e^{(2-1) / 3}\right) \\
= & 0.4744+0.3322+0.9165=1.7231
\end{aligned}
$$




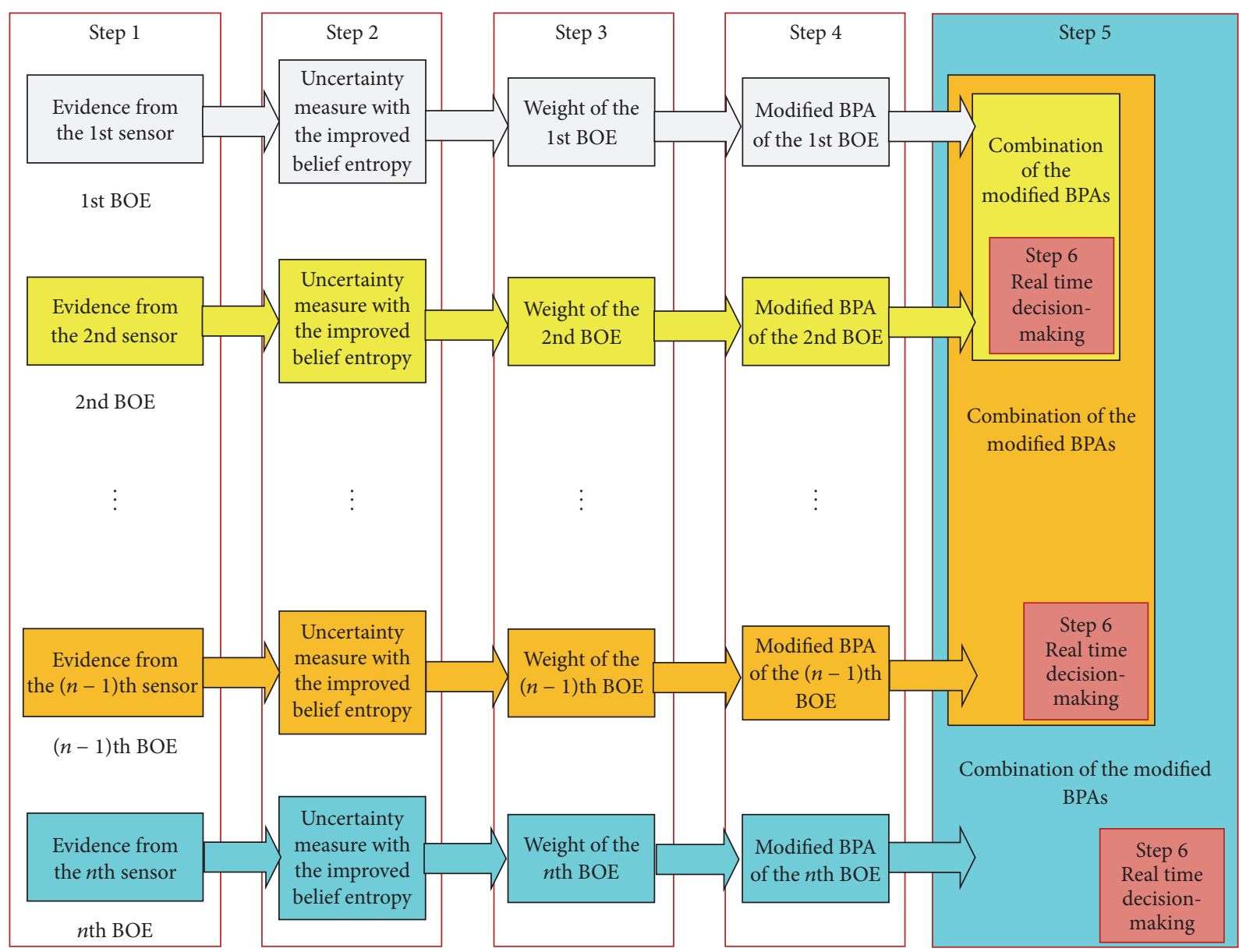

FIGURE 2: The flowchart of the proposed approach for decision-making.

$$
\begin{aligned}
E_{I d}\left(m_{5}\right)= & -\sum_{A \subseteq X} m_{5}(A) \log _{2}\left(\frac{m_{5}(A)}{2^{|A|}-1} e^{(|A|-1) /|X|}\right) \\
= & -0.6 \log _{2}\left(\frac{0.6}{2^{1}-1} e^{(1-1) / 3}\right) \\
& -0.1 \log _{2}\left(\frac{0.1}{2^{1}-1} e^{(1-1) / 3}\right) \\
& -0.3 \log _{2}\left(\frac{0.3}{2^{2}-1} e^{(2-1) / 3}\right) \\
= & 0.4422+0.3322+0.8523=1.6267 .
\end{aligned}
$$

Intuitively, the second BOE $m_{2}$ is conflicting with the other BOEs; this is also successfully indicated by the abnormal value of the improved belief entropy. In other words, the improved belief entropy value of $m_{2}$, which is 0.4690 , is abnormal in comparison with that of the other four BOEs.

4.3. Decision-Making Procedures. In real applications, for example, air battle, the real-time requirement is highly concerned, so decision-making in target recognition needs to be finished in real time. If decision-making needs to be finished in real time, then data fusion needs to be processed instantly with the upcoming sensor report. The procedures for decision-making based on the improved belief entropy are designed in Figure 2; six steps are needed as follows.

Step 1. Evidence from sensor report is modeled as the BOE.

An example of this step is given in Section 4.1; each piece of evidence is modeled as a BOE.

Step 2. Uncertainty measure of each BOE with the improved belief entropy.

Generally, the more dispersive the mass value is assigned among the power set, the bigger the new belief entropy of the $\mathrm{BOE}$ will be. An illustrative example of this step is shown in Section 4.2; the proposed belief entropy is used to measure the uncertain degree of each BOE in Section 4.1.

Step 3. Relative weight of the incoming BOEs is calculated based on the improved uncertainty measure.

A big entropy value corresponds to a big information volume. It is commonly accepted that the bigger the entropy is, the higher the uncertain degree will be. The relative weight 
of each BOE is defined as the relative weight of the new belief entropy. For the $i$ th $\mathrm{BOE}$, its relative weight among all the available $n$ BOEs, denoted as $W_{\mathrm{BOE}}\left(m_{i}\right)$, is defined as follows:

$$
W_{\mathrm{BOE}}\left(m_{i}\right)=\frac{E_{I d}\left(m_{i}\right)}{\sum_{j=1}^{n} E_{I d}\left(m_{j}\right)} .
$$

The relative weight of each BOE in Section 4.1 can be calculated with (18); the results are shown as follows:

$$
\begin{aligned}
& W_{\mathrm{BOE}}\left(m_{1}\right)=\frac{E_{I d}\left(m_{1}\right)}{\sum_{j=1}^{5} E_{I d}\left(m_{j}\right)} \\
& =\frac{1.5664}{1.5664+0.4690+1.6409+1.7231+1.6267} \\
& \quad=0.2229, \\
& W_{\mathrm{BOE}}\left(m_{2}\right)=\frac{E_{I d}\left(m_{2}\right)}{\sum_{j=1}^{5} E_{I d}\left(m_{j}\right)} \\
& =\frac{0.4690}{1.5664+0.4690+1.6409+1.7231+1.6267} \\
& =0.0668,
\end{aligned}
$$

$$
\begin{aligned}
& W_{\text {BOE }}\left(m_{3}\right)=\frac{E_{I d}\left(m_{3}\right)}{\sum_{j=1}^{5} E_{I d}\left(m_{j}\right)} \\
& =\frac{1.6409}{1.5664+0.4690+1.6409+1.7231+1.6267} \\
& =0.2335,
\end{aligned}
$$$$
W_{\mathrm{BOE}}\left(m_{4}\right)=\frac{E_{I d}\left(m_{4}\right)}{\sum_{j=1}^{5} E_{I d}\left(m_{j}\right)}
$$$$
=\frac{1.7231}{1.5664+0.4690+1.6409+1.7231+1.6267}
$$$$
=0.2452 \text {, }
$$$$
W_{\mathrm{BOE}}\left(m_{5}\right)=\frac{E_{I d}\left(m_{5}\right)}{\sum_{j=1}^{5} E_{I d}\left(m_{j}\right)}
$$$$
=\frac{1.6267}{1.5664+0.4690+1.6409+1.7231+1.6267}
$$$$
=0.2315 \text {. }
$$

Step 4. The modified BPAs are derived for sensor data fusion.

For a proposition $A$, the modified BPA based on $n$ available BOEs is defined as follows:

$$
m(A)=\sum_{i=1}^{n} m_{i}(A) W_{\mathrm{BOE}}\left(m_{i}\right) .
$$

For example, with (20), the BPAs in Section 4.1 are modified with the relative weight of each $\mathrm{BOE}$; the results are shown as follows:

$$
\begin{aligned}
m(\{a\})= & 0.41 W_{\mathrm{BOE}}\left(m_{1}\right)+0.58 W_{\mathrm{BOE}}\left(m_{3}\right) \\
& +0.55 W_{\mathrm{BOE}}\left(m_{4}\right)+0.6 W_{\mathrm{BOE}}\left(m_{5}\right) \\
= & 0.5007 \\
m(\{b\})= & 0.29 W_{\mathrm{BOE}}\left(m_{1}\right)+0.9 W_{\mathrm{BOE}}\left(m_{2}\right) \\
& +0.07 W_{\mathrm{BOE}}\left(m_{3}\right)+0.1 W_{\mathrm{BOE}}\left(m_{4}\right) \\
& +0.1 W_{\mathrm{BOE}}\left(m_{5}\right)=0.1888 \\
m(\{c\})= & 0.3 W_{\mathrm{BOE}}\left(m_{1}\right)+0.1 W_{\mathrm{BOE}}\left(m_{2}\right)=0.0736 \\
m(\{a, c\})= & 0.35 W_{\mathrm{BOE}}\left(m_{3}\right)+0.35 W_{\mathrm{BOE}}\left(m_{4}\right) \\
& +0.3 W_{\mathrm{BOE}}\left(m_{5}\right)=0.2370 .
\end{aligned}
$$

Step 5. The weighted BPAs are fused with Dempster's rule of combination in (5)-(6) by $(n-1)$ time $(s)$.

For example, the modified BPAs in Step 4 are fused with Dempster's rule of combination by four times; the fused results are shown as follows:

$$
\begin{aligned}
m(\{a\}) & =((((m \oplus m) \oplus m) \oplus m) \oplus m)(\{a\}) \\
& =0.9873 \\
m(\{b\}) & =((((m \oplus m) \oplus m) \oplus m) \oplus m)(\{b\}) \\
& =0.0011 \\
m(\{c\}) & =((((m \oplus m) \oplus m) \oplus m) \oplus m)(\{c\}) \\
& =0.0097 \\
m(\{a, c\}) & =((((m \oplus m) \oplus m) \oplus m) \oplus m)(\{a, c\}) \\
& =0.0034 .
\end{aligned}
$$

Step 6. Real-time decision-making based on the fused results.

For example, taking into consideration the target recognition problem expressed in Section 4.1, with the aforementioned five steps, now it is safe to make the conclusion that $a$ is the recognized target.

Based on the aforementioned six steps, the decisionmaking results corresponding to two, three, four, and five BOEs are shown in Table 4, respectively. It can be concluded that with the proposed method the right decision will be made even if there are only three BOEs, because the belief on the proposition $\{a\}$ is more than $80 \%$ in the fusion result with three BOEs. The inference of the wrong report $m_{2}$ can be overcome instantly with the incoming sensor reports. The fusion results with all the five sensor reports will have a belief of over $98 \%$ on the right recognized target $a$.

4.4. Discussion. In order to test the efficiency of the proposed method, the fusion results are compared with the other methods in [78-80]. The results of different combination methods addressed on this issue are shown in Table 5. The comparisons 
TABLE 4: Decision-making results based on sensor data fusion.

\begin{tabular}{|c|c|c|}
\hline BOEs & Fusion results & Recognition result \\
\hline \multirow{4}{*}{$m_{1}, m_{2}$} & $m(\{a\})=0.2849$ & \multirow{4}{*}{$\begin{array}{l}\text { Uncertain; no proposition } \\
\text { has a belief over } 60.00 \%\end{array}$} \\
\hline & $m(\{b\})=0.5305$ & \\
\hline & $m(\{c\})=0.1845$ & \\
\hline & $m(\{a, c\})=0$ & \\
\hline \multirow{4}{*}{$m_{1}, m_{2}, m_{3}$} & $m(\{a\})=0.8148$ & \multirow{4}{*}{$a$, with a belief of $81.48 \%$} \\
\hline & $m(\{b\})=0.0794$ & \\
\hline & $m(\{c\})=0.0904$ & \\
\hline & $m(\{a, c\})=0.0154$ & \\
\hline \multirow{4}{*}{$m_{1}, m_{2}, m_{3}, m_{4}$} & $m(\{a\})=0.9495$ & \multirow{4}{*}{$a$, with a belief of $94.95 \%$} \\
\hline & $m(\{b\})=0.0092$ & \\
\hline & $m(\{c\})=0.0317$ & \\
\hline & $m(\{a, c\})=0.0096$ & \\
\hline \multirow{4}{*}{$m_{1}, m_{2}, m_{3}, m_{4}, m_{5}$} & $m(\{a\})=0.9873$ & \multirow{4}{*}{$a$, with a belief of $98.73 \%$} \\
\hline & $m(\{b\})=0.0011$ & \\
\hline & $m(\{c\})=0.0097$ & \\
\hline & $m(\{a, c\})=0.0034$ & \\
\hline
\end{tabular}

TABLE 5: Fusion results with different combination rules.

\begin{tabular}{|c|c|c|c|c|}
\hline & $m_{1}, m_{2}$ & $m_{1}, m_{2}, m_{3}$ & $m_{1}, m_{2}, m_{3}, m_{4}$ & $m_{1}, m_{2}, m_{3}, m_{4}, m_{5}$ \\
\hline \multirow{4}{*}{ Dempster's rule [20] } & $m(\{a\})=0$ & $m(\{a\})=0$ & $m(\{a\})=0$ & $m(\{a\})=0$ \\
\hline & $m(\{b\})=0.8969$ & $m(\{b\})=0.6575$ & $m(\{b\})=0.3321$ & $m(\{b\})=0.1422$ \\
\hline & $m(\{c\})=0.1031$ & $m(\{c\})=0.3425$ & $m(\{c\})=0.6679$ & $m(\{c\})=0.8578$ \\
\hline & $m(\{a, c\})=0$ & $m(\{a, c\})=0$ & $m(\{a, c\})=0$ & $m(\{a, c\})=0$ \\
\hline \multirow{5}{*}{ Yager's rule [76] } & $m(\{a\})=0$ & $m(\{a\})=0.4112$ & $m(\{a\})=0.6508$ & $m(\{a\})=0.7732$ \\
\hline & $m(\{b\})=0.2610$ & $m(\{b\})=0.0679$ & $m(\{b\})=0.0330$ & $m(\{b\})=0.0167$ \\
\hline & $m(\{c\})=0.0300$ & $m(\{c\})=0.0105$ & $m(\{c\})=0.0037$ & $m(\{c\})=0.0011$ \\
\hline & $m(\{a, c\})=0$ & $m(\{a, c\})=0.2481$ & $m(\{a, c\})=0.1786$ & $m(\{a, c\})=0.0938$ \\
\hline & $m(\{X\})=0.7090$ & $m(\{X\})=0.2622$ & $m(\{X\})=0.1339$ & $m(\{X\})=0.1152$ \\
\hline \multirow{4}{*}{ Murphy's rule [77] } & $m(\{a\})=0.0964$ & $m(\{a\})=0.4619$ & $m(\{a\})=0.8362$ & $m(\{a\})=0.9620$ \\
\hline & $m(\{b\})=0.8119$ & $m(\{b\})=0.4497$ & $m(\{b\})=0.1147$ & $m(\{b\})=0.0210$ \\
\hline & $m(\{c\})=0.0917$ & $m(\{c\})=0.0794$ & $m(\{c\})=0.0410$ & $m(\{c\})=0.0138$ \\
\hline & $m(\{a, c\})=0$ & $m(\{a, c\})=0.0090$ & $m(\{a, c\})=0.0081$ & $m(\{a, c\})=0.0032$ \\
\hline \multirow{4}{*}{ Deng et al's method [78] } & $m(\{a\})=0.0964$ & $m(\{a\})=0.4974$ & $m(\{a\})=0.9089$ & $m(\{a\})=0.9820$ \\
\hline & $m(\{b\})=0.8119$ & $m(\{b\})=0.4054$ & $m(\{b\})=0.0444$ & $m(\{b\})=0.0039$ \\
\hline & $m(\{c\})=0.0917$ & $m(\{c\})=0.0888$ & $m(\{c\})=0.0379$ & $m(\{c\})=0.0107$ \\
\hline & $m(\{a, c\})=0$ & $m(\{a, c\})=0.0084$ & $m(\{a, c\})=0.0089$ & $m(\{a, c\})=0.0034$ \\
\hline \multirow{4}{*}{ Zhang et al's method [79] } & $m(\{a\})=0.0964$ & $m(\{a\})=0.5681$ & $m(\{a\})=0.9142$ & $m(\{a\})=0.9820$ \\
\hline & $m(\{b\})=0.8119$ & $m(\{b\})=0.3319$ & $m(\{b\})=0.0395$ & $m(\{b\})=0.0034$ \\
\hline & $m(\{c\})=0.0917$ & $m(\{c\})=0.0929$ & $m(\{c\})=0.0399$ & $m(\{c\})=0.0115$ \\
\hline & $m(\{a, c\})=0$ & $m(\{a, c\})=0.0084$ & $m(\{a, c\})=0.0083$ & $m(\{a, c\})=0.0032$ \\
\hline \multirow{4}{*}{ Yuan et al's method [80] } & $m(\{a\})=0.2678$ & $m(\{a\})=0.8274$ & $m(\{a\})=0.9596$ & $m(\{a\})=0.9886$ \\
\hline & $m(\{b\})=0.5552$ & $m(\{b\})=0.0609$ & $m(\{b\})=0.0032$ & $m(\{b\})=0.0002$ \\
\hline & $m(\{c\})=0.1770$ & $m(\{c\})=0.0986$ & $m(\{c\})=0.0267$ & $m(\{c\})=0.0072$ \\
\hline & $m(\{a, c\})=0$ & $m(\{a, c\})=0.0131$ & $m(\{a, c\})=0.0106$ & $m(\{a, c\})=0.0039$ \\
\hline \multirow{4}{*}{ The proposed method } & $m(\{a\})=0.2849$ & $m(\{a\})=0.8148$ & $m(\{a\})=0.9495$ & $m(\{a\})=0.9873$ \\
\hline & $m(\{b\})=0.5305$ & $m(\{b\})=0.0794$ & $m(\{b\})=0.0092$ & $m(\{b\})=0.0011$ \\
\hline & $m(\{c\})=0.1845$ & $m(\{c\})=0.0904$ & $m(\{c\})=0.0317$ & $m(\{c\})=0.0097$ \\
\hline & $m(\{a, c\})=0$ & $m(\{a, c\})=0.0154$ & $m(\{a, c\})=0.0096$ & $m(\{a, c\})=0.0034$ \\
\hline
\end{tabular}




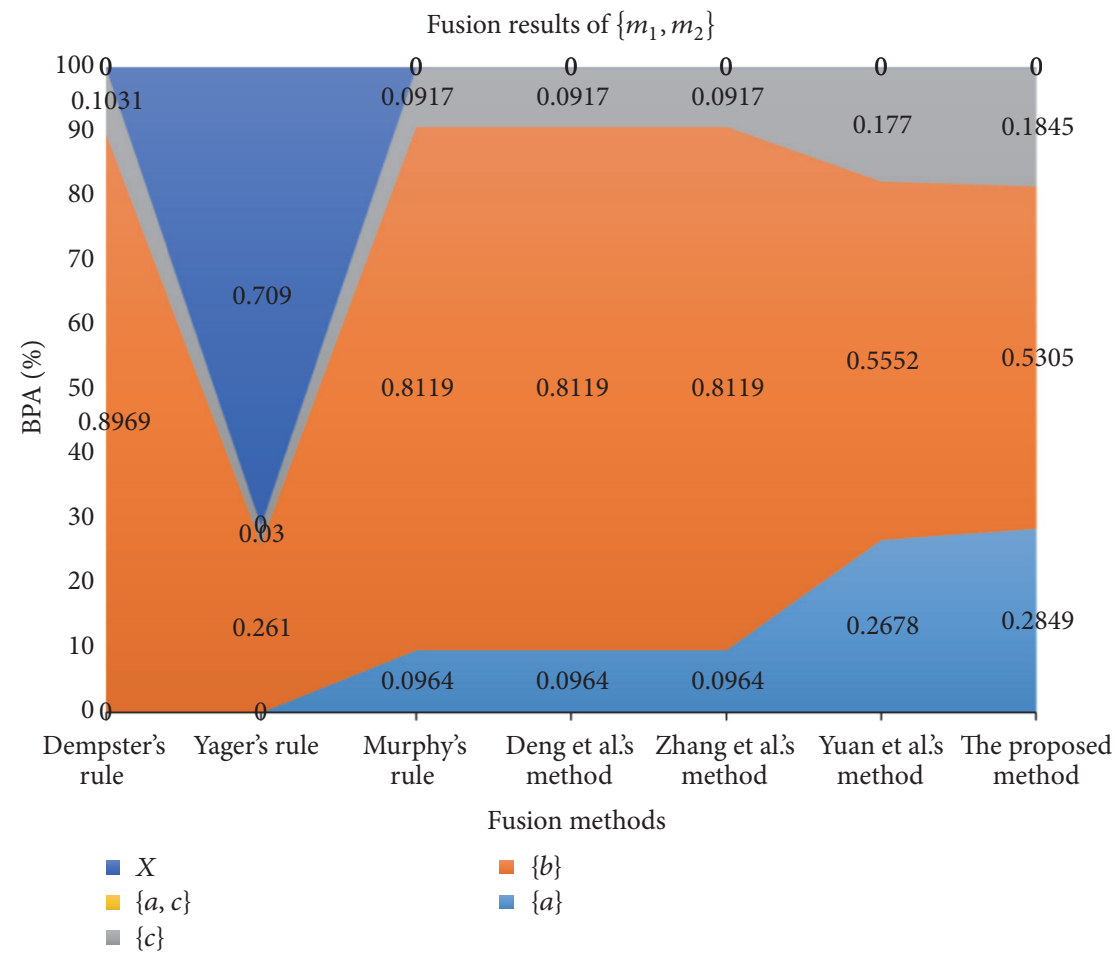

FIgURE 3: Fusion results of two BOEs.

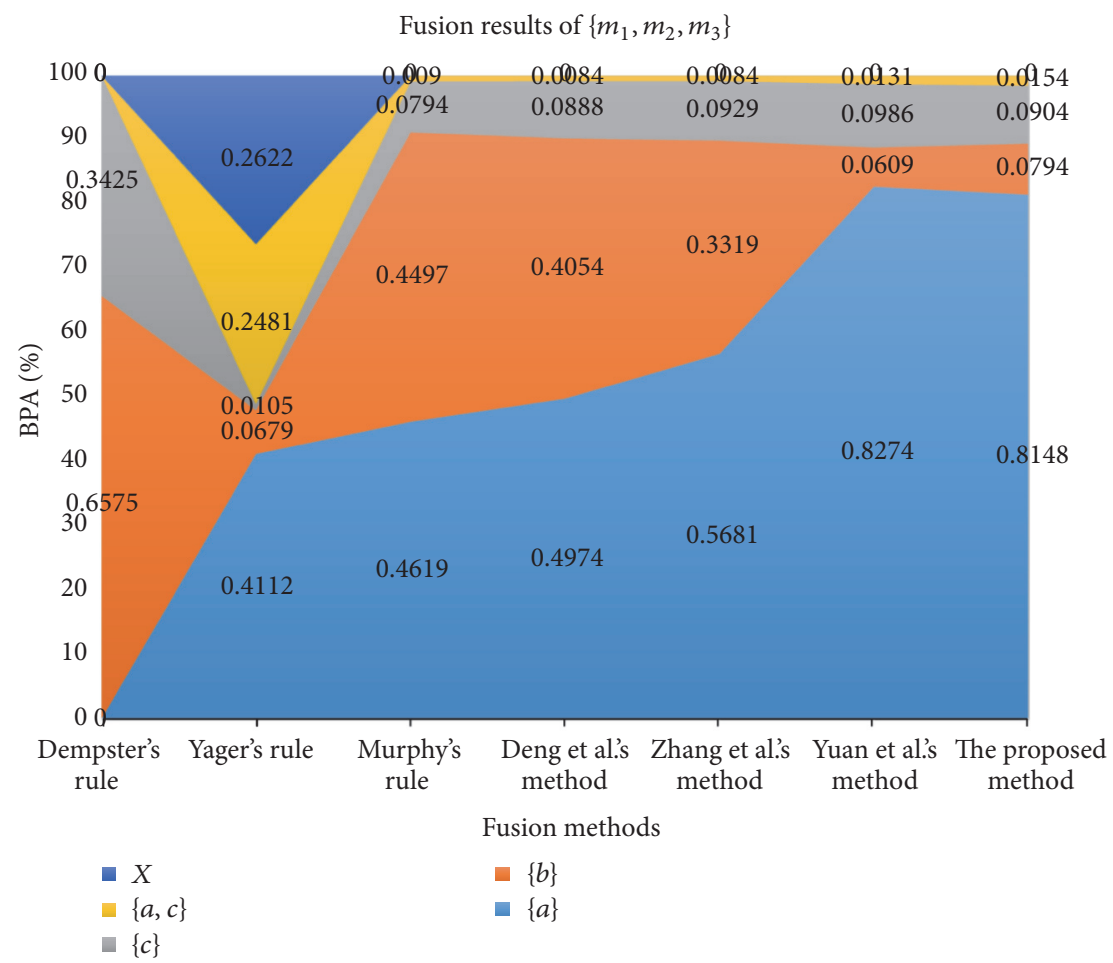

FIGURE 4: Fusion results of three BOEs.

of the fusion results with different fusion methods of two, three, four, and five BOEs are shown in Figures 3, 4, 5, and 6 , respectively.
Figure 3 shows the fusion results with two BOEs. In this case, one cannot make a decision with Yager's combination rule because it assigns most belief to the universal set, while 


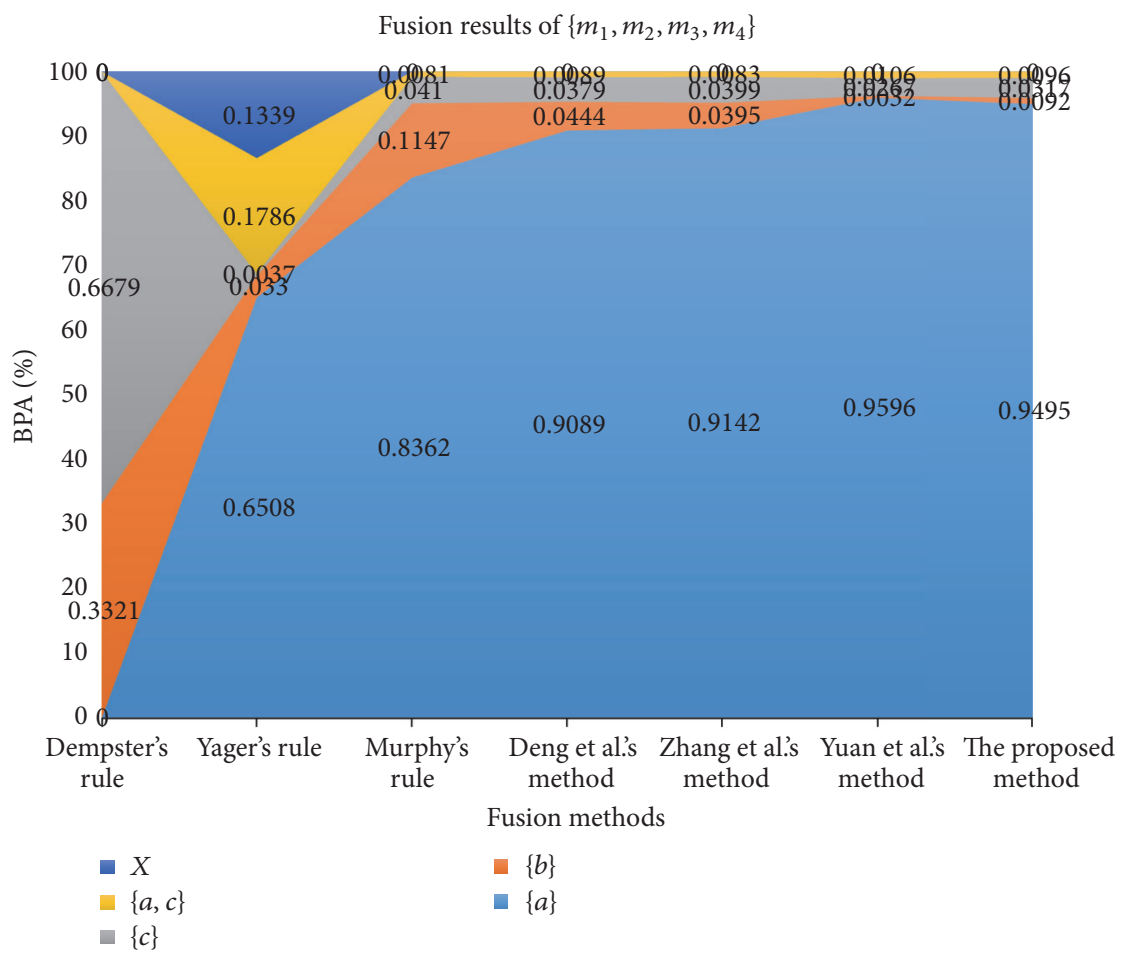

FIgURE 5: Fusion results of four BOEs.

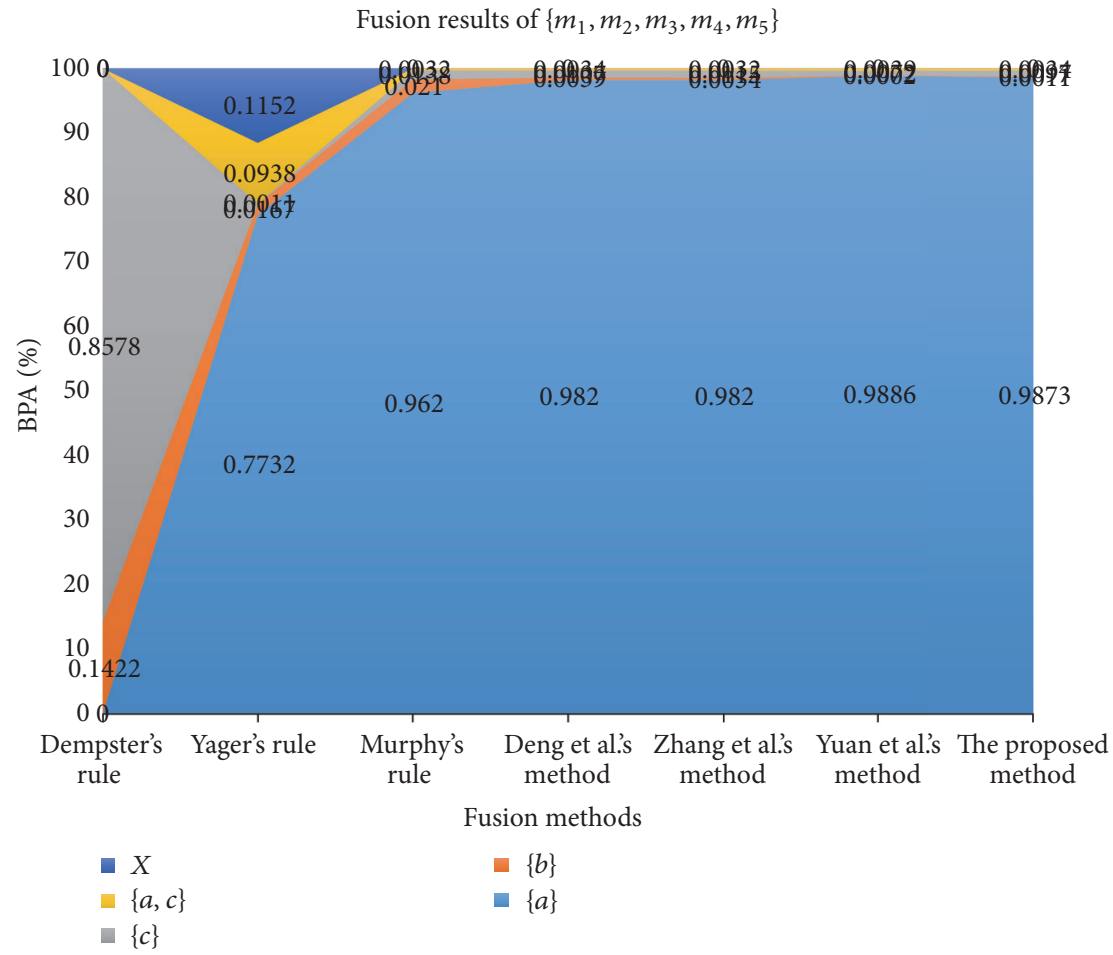

FIGURE 6: Fusion results of five BOEs.

the other methods except for the proposed method and Yuan et al.s method have quite a high belief on the object $b$; this is in conflict with the reality because the second $\mathrm{BOE} m_{2}$ comes from a bad sensor and $b$ cannot be the target.
Figure 4 shows the fusion results with three BOEs. The fusion result with Dempster's rule is counterintuitive. The fusion results with Yager's rule, Murphy's rule, and Deng et al.'s and Zhang et al's methods have limited belief on 
the right target $b$. Even if there are only three BOEs, the proposed method and Yuan et al's method can make the decision that $b$ is the target with a belief of more than $80 \%$.

Figure 5 shows the fusion results with four BOEs. When it comes to three of four BOEs to support that the target is $b$, the fusion result with Dempster's rule still leads to the wrong conclusion due to the conflicting evidence in the second BOE $m_{2}$, while the proposed method and Yuan et al.s method have much more belief on the target $b$ than the other methods.

The performance of different methods shown in Figure 6 is similar to that in Figure 5. With all the five sensor reports, both the proposed method and Yuan et al.s method will have a belief of over 98\% on the recognized target a.

It seems that the performance of the proposed method is similar to Yuan et al.s method [80]. However, the proposed method has at least three aspects of superiorities in comparison with Yuan et al.s method. Firstly, the improved belief entropy has an improved capacity of uncertainty measure compared to the Deng entropy used in Yuan et al.s method. According to the discussion related to Example 1 in Sections 3.1 and 3.2, the proposed belief entropy can effectively measure the different uncertain degree among different BOEs even if the same mass value is assigned on different FODs, while both Deng entropy and the weighted Hartley entropy are failed. Secondly, both the support degree and the information volume of each evidence defined in [80] are based on the mass function, which means that the BOEs are used twice to define these two indexes; however, the relation of these two indexes is not clear, for example, the coupling relation. Thirdly, the proposed method is only based on the information volume measured by the improved belief entropy; its physical meaning is clearer than that in Yuan et al.s method. Above all, the proposed method is more reasonable and efficient than the other methods.

A few reasons contribute to the effectiveness of the proposed decision-making approach. First of all, the sensor data is preprocessed properly before applying the combination rules. This is very important in sensor data fusion especially if there is conflict evidence. Secondly, the weights of BOEs are calculated based on the proposed belief entropy. The effectiveness and superiority of the new belief entropy verified in Section 3.3 ensure the rationality and good performance of the proposed decision-making approach. Finally, the final fused rule is based on Dempster's rule of combination. The merits of Dempster's rule, such as satisfying the commutativity and associativity, guarantee the rationality of the fusion result.

\section{Conclusions}

In this paper, an improved belief entropy is proposed. The new belief entropy improves the performance of Deng entropy and some other uncertainty measures in DempsterShafer framework. The new belief entropy considers the uncertain information consisted in not only the mass function and the cardinality of the proposition, but also the scale of the FOD and the relative scale of each proposition with respect to the FOD. Numerical examples show that the new belief entropy can quantify the uncertain degree of the BOE more accurately than the other uncertainty measures.

A new decision-making approach is presented in this paper and applied to a case study. The new uncertainty measure-based decision-making approach shows the efficiency and merit of the new belief entropy. In the following researches, the proposed belief entropy will be used to solve more problems related to uncertain information processing in real world applications.

\section{Competing Interests}

The authors declare that there is no conflict of interests regarding the publication of this paper.

\section{Acknowledgments}

The work is partially supported by National Natural Science Foundation of China (Grant no. 61671384), Natural Science Basic Research Plan in Shaanxi Province of China (Grant no. 2016JM6018), the Fund of SAST (Grant no. SAST2016083), and Innovation Foundation for Doctor Dissertation of Northwestern Polytechnical University.

\section{References}

[1] E. Šiožinyte, J. Antuchevičiene, and V. Kutut, "Upgrading the old vernacular building to contemporary norms: multiple criteria approach," Journal of Civil Engineering \& Management, vol. 20, no. 2, pp. 291-298, 2014.

[2] J. Antucheviciene, Z. Kala, M. Marzouk, and E. R. Vaidogas, "Solving civil engineering problems by means of fuzzy and stochastic mcdm methods: current state and future research," Mathematical Problems in Engineering, vol. 2015, Article ID 362579, 16 pages, 2015.

[3] E. K. Zavadskas, J. Antucheviciene, S. H. Razavi Hajiagha, and S. S. Hashemi, "The interval-valued intuitionistic fuzzy MULTIMOORA method for group decision making in engineering," Mathematical Problems in Engineering, vol. 2015, Article ID 560690, 13 pages, 2015.

[4] M. A. Sodenkamp, M. Tavana, and D. Di Caprio, "Modeling synergies in multi-criteria supplier selection and order allocation: an application to commodity trading," European Journal of Operational Research, vol. 254, no. 3, pp. 859-874, 2016.

[5] M. K. Ghorabaee, E. K. Zavadskas, M. Amiri, and J. Antucheviciene, "A new method of assessment based on fuzzy ranking and aggregated weights (AFRAW) for MCDM problems under type-2 fuzzy environment," Economic Computation \& Economic Cybernetics Studies \& Research, vol. 50, pp. 39-68, 2016.

[6] T. Aven, "Supplementing quantitative risk assessments with a stage addressing the risk understanding of the decision maker," Reliability Engineering and System Safety, vol. 152, pp. 51-57, 2016.

[7] T. Aven and E. Zio, "Some considerations on the treatment of uncertainties in risk assessment for practical decision making," 
Reliability Engineering and System Safety, vol. 96, no. 1, pp. 6474, 2011.

[8] H. Ahmadi, M. Nilashi, and O. Ibrahim, "Organizational decision to adopt hospital information system: an empirical investigation in the case of Malaysian public hospitals," International Journal of Medical Informatics, vol. 84, no. 3, pp. 166-188, 2015.

[9] M. Nilashi, H. Ahmadi, A. Ahani, R. Ravangard, and O. B. Ibrahim, "Determining the importance of hospital information system adoption factors using fuzzy Analytic Network Process (ANP)," Technological Forecasting \& Social Change, vol. 111, pp. 244-264, 2016.

[10] M. J. North, C. M. Macal, J. St. Aubin et al., "Multiscale agentbased consumer market modeling," Complexity, vol. 15, no. 5, pp. 37-47, 2010.

[11] E. K. Zavadskas, R. Baušys, D. Stanujkic, and M. MagdalinovicKalinovic, "Selection of lead-zinc flotation circuit design by applying WASPAS method with single-valued neutrosophic set," Acta Montanistica Slovaca, vol. 21, no. 2, pp. 85-92, 2016.

[12] E. K. Zavadskas, R. Baušys, and M. Lazauskas, "Sustainable assessment of alternative sites for the construction of a waste incineration plant by applying WASPAS method with singlevalued neutrosophic set," Sustainability, vol. 7, no. 12, pp. 1592315936, 2015.

[13] W. Feller, An Introduction to Probability Theory and Its Applications, John Wiley \& Sons, New York, NY, USA, 2nd edition, 1957.

[14] Z.-S. Chen, K.-S. Chin, Y.-L. Li, and Y. Yang, "Proportional hesitant fuzzy linguistic term set for multiple criteria group decision making," Information Sciences, vol. 357, pp. 61-87, 2016.

[15] L. A. Zadeh, "Fuzzy sets," Information and Control, vol. 8, no. 3, pp. 338-353, 1965.

[16] K. Khalili-Damghani, M. Tavana, and M. Amirkhan, "A fuzzy bi-objective mixed-integer programming method for solving supply chain network design problems under ambiguous and vague conditions," International Journal of Advanced Manufacturing Technology, vol. 73, no. 9-12, pp. 1567-1595, 2014.

[17] F. Sabahi and M.-R. Akbarzadeh-T, "Introducing validity in fuzzy probability for judicial decision-making," International Journal of Approximate Reasoning, vol. 55, no. 6, pp. 1383-1403, 2014.

[18] X. Wang, J. Zhu, Y. Song, and L. Lei, "Combination of unreliable evidence sources in intuitionistic fuzzy MCDM framework," Knowledge-Based Systems, vol. 97, pp. 24-39, 2016.

[19] A. P. Dempster, "Upper and lower probabilities induced by a multivalued mapping," Annals of Mathematical Statistics, vol. 38, pp. 325-339, 1967.

[20] G. Shafer, A Mathematical Theory of Evidence, Princeton University Press, Princeton, NJ, USA, 1976.

[21] R. R. Yager, "Decision making using minimization of regret," International Journal of Approximate Reasoning, vol. 36, no. 2, pp. 109-128, 2004.

[22] C. Fu, J.-B. Yang, and S.-L. Yang, "A group evidential reasoning approach based on expert reliability," European Journal of Operational Research, vol. 246, no. 3, pp. 886-893, 2015.

[23] Z. Pawlak, "Rough sets," International Journal of Computer \& Information Sciences, vol. 11, no. 5, pp. 341-356, 1982.

[24] B. Sun, W. Ma, and H. Zhao, "An approach to emergency decision making based on decision-theoretic rough set over two universes," Soft Computing, vol. 20, no. 9, pp. 3617-3628, 2016.
[25] C. Luo, T. Li, Z. Yi, and H. Fujita, "Matrix approach to decision-theoretic rough sets for evolving data," KnowledgeBased Systems, vol. 99, pp. 123-134, 2016.

[26] C. Fu and D. L. Xu, "Determining attribute weights to improve solution reliability and its application to selecting leading industries," Annals of Operations Research, vol. 245, pp. 401-426, 2014.

[27] J. Wang, Y. Hu, F. Xiao, X. Deng, and Y. Deng, "A novel method to use fuzzy soft sets in decision making based on ambiguity measure and Dempster-Shafer theory of evidence: an application in medical diagnosis," Artificial Intelligence in Medicine, vol. 69, pp. 1-11, 2016.

[28] X. Deng, X. Zheng, X. Su et al., "An evidential game theory framework in multi-criteria decision making process," Applied Mathematics and Computation, vol. 244, pp. 783-793, 2014.

[29] B. Kang, Y. Hu, Y. Deng, and D. Zhou, "A new methodology of multicriteria decision-making in supplier selection based on Z-numbers," Mathematical Problems in Engineering, vol. 2016, Article ID 8475987, 17 pages, 2016.

[30] Z.-G. Liu, Q. Pan, and J. Dezert, "A new belief-based K-nearest neighbor classification method," Pattern Recognition, vol. 46, no. 3, pp. 834-844, 2013.

[31] J. Ma, W. Liu, P. Miller, and H. Zhou, "An evidential fusion approach for gender profiling," Information Sciences, vol. 333, pp. 10-20, 2016.

[32] Z.-G. Liu, Q. Pan, J. Dezert, and G. Mercier, "Credal c-means clustering method based on belief functions," Knowledge-Based Systems, vol. 74, pp. 119-132, 2015.

[33] D. Han, W. Liu, J. Dezert, and Y. Yang, "A novel approach to pre-extracting support vectors based on the theory of belief functions," Knowledge-Based Systems, vol. 110, pp. 210-223, 2016.

[34] Z.-G. Liu, Q. Pan, J. Dezert, and A. Martin, "Adaptive imputation of missing values for incomplete pattern classification," Pattern Recognition, vol. 52, pp. 85-95, 2016.

[35] X. Su, Y. Deng, S. Mahadevan, and Q. Bao, "An improved method for risk evaluation in failure modes and effects analysis of aircraft engine rotor blades," Engineering Failure Analysis, vol. 26, pp. 164-174, 2012.

[36] W. Jiang, C. Xie, M. Zhuang, Y. Shou, and Y. Tang, "Sensor data fusion with z-numbers and its application in fault diagnosis," Sensors, vol. 16, no. 9, p. 1509, 2016.

[37] K. Yuan, F. Xiao, L. Fei, B. Kang, and Y. Deng, "Modeling sensor reliability in fault diagnosis based on evidence theory," Sensors, vol. 16, no. 1, article 113, 2016.

[38] W. Jiang, B. Wei, X. Qin, J. Zhan, and Y. Tang, "Sensor data fusion based on a new conflict measure," Mathematical Problems in Engineering, vol. 2016, Article ID 5769061, 11 pages, 2016.

[39] K.-S. Chin, C. Fu, and Y. Wang, "A method of determining attribute weights in evidential reasoning approach based on incompatibility among attributes," Computers and Industrial Engineering, vol. 87, pp. 150-162, 2015.

[40] W. S. Du and B. Q. Hu, "Attribute reduction in ordered decision tables via evidence theory," Information Sciences, vol. 364-365, pp. 91-110, 2016.

[41] C. Fu and Y. Wang, "An interval difference based evidential reasoning approach with unknown attribute weights and utilities of assessment grades," Computers \& Industrial Engineering, vol. 81, pp. 109-117, 2015.

[42] Y.-M. Wang and T. M. S. Elhag, "A comparison of neural network, evidential reasoning and multiple regression analysis 
in modelling bridge risks," Expert Systems with Applications, vol. 32, no. 2, pp. 336-348, 2007.

[43] W. Jiang, C. Xie, B. Wei, and D. Zhou, "A modified method for risk evaluation in failure modes and effects analysis of aircraft turbine rotor blades," Advances in Mechanical Engineering, vol. 8, no. 4, pp. 1-16, 2016.

[44] R. R. Yager and D. P. Filev, "Including probabilistic uncertainty in fuzzy logic controller modeling using dempster-shafer theory," IEEE Transactions on Systems, Man, and Cybernetics, vol. 25, no. 8, pp. 1221-1230, 1995.

[45] Y. Tang, D. Zhou, and W. Jiang, "A new fuzzy-evidential controller for stabilization of the planar inverted pendulum system," PLoS ONE, vol. 11, no. 8, Article ID e0160416, 2016.

[46] Y.-M. Wang, J.-B. Yang, D.-L. Xu, and K.-S. Chin, "Consumer preference prediction by using a hybrid evidential reasoning and belief rule-based methodology," Expert Systems with Applications, vol. 36, no. 4, pp. 8421-8430, 2009.

[47] J. Ma, W. Liu, and S. Benferhat, "A belief revision framework for revising epistemic states with partial epistemic states," International Journal of Approximate Reasoning, vol. 59, pp. 20-40, 2015.

[48] K. Zhou, A. Martin, Q. Pan, and Z.-G. Liu, "Median evidential c-means algorithm and its application to community detection," Knowledge-Based Systems, vol. 74, pp. 69-88, 2015.

[49] L. A. Zadeh, "Simple view of the dempster-shafer theory of evidence and its implication for the rule of combination," $A I$ Magazine, vol. 7, no. 2, pp. 85-90, 1986.

[50] W. Liu, "Analyzing the degree of conflict among belief functions," Artificial Intelligence, vol. 170, no. 11, pp. 909-924, 2006.

[51] J. Schubert, "Conflict management in Dempster-Shafer theory using the degree of falsity," International Journal of Approximate Reasoning, vol. 52, no. 3, pp. 449-460, 2011.

[52] Y. Deng, "Generalized evidence theory," Applied Intelligence, vol. 43, no. 3, pp. 530-543, 2015.

[53] X. Su, S. Mahadevan, W. Han, and Y. Deng, "Combining dependent bodies of evidence," Applied Intelligence, vol. 44, no. 3, pp. 634-644, 2016.

[54] X. Su, S. Mahadevan, P. Xu, and Y. Deng, "Dependence assessment in human reliability analysis using evidence theory and AHP," Risk Analysis, vol. 35, no. 7, pp. 1296-1316, 2015.

[55] X. Su, S. Mahadevan, P. Xu, and Y. Deng, "Handling of dependence in Dempster-Shafer theory," International Journal of Intelligent Systems, vol. 30, no. 4, pp. 441-467, 2015.

[56] X. Deng, Q. Liu, Y. Deng, and S. Mahadevan, "An improved method to construct basic probability assignment based on the confusion matrix for classification problem," Information Sciences, vol. 340-341, pp. 250-261, 2016.

[57] Y. Yi and Y. Liu, "Iterative approximation of basic belief assignment based on distance of evidence," PLoS ONE, vol. 11, no. 2, Article ID e0147799, 2016.

[58] W. Jiang, J. Zhan, D. Zhou, and X. Li, "A method to determine generalized basic probability assignment in the open world," Mathematical Problems in Engineering, vol. 2016, Article ID 3878634, 11 pages, 2016.

[59] P. Smets and R. Kennes, "The transferable belief model," Artificial Intelligence, vol. 66, no. 2, pp. 191-234, 1994.

[60] P. Smets, "Belief functions on real numbers," International Journal of Approximate Reasoning, vol. 40, no. 3, pp. 181-223, 2005.
[61] D. Zhou, Y. Tang, and W. Jiang, "A modified model of failure mode and effects analysis based on generalized evidence theory," Mathematical Problems in Engineering, vol. 2016, Article ID 4512383, 11 pages, 2016.

[62] C. E. Shannon, "A mathematical theory of communication," ACM SIGMOBILE Mobile Computing and Communications Review, vol. 5, no. 1, pp. 3-55, 2001.

[63] M. Tavana, M. A. Sodenkamp, and M. Pirdashti, "A fuzzy opportunity and threat aggregation approach in multicriteria decision analysis," Fuzzy Optimization and Decision Making, vol. 9, no. 4, pp. 455-492, 2010.

[64] T. Dejus and J. Antuchevičiene, "Assessment of health and safety solutions at a construction site," Journal of Civil Engineering and Management, vol. 19, no. 5, pp. 728-737, 2013.

[65] M. Tavana, K. Khalili-Damghani, and R. Rahmatian, "A hybrid fuzzy MCDM method for measuring the performance of publicly held pharmaceutical companies," Annals of Operations Research, vol. 226, pp. 589-621, 2015.

[66] U. Hohle, "Entropy with respect to plausibility measures," in Proceedings of the 12th IEEE International Symposium on Multiple-Valued Logic (MVL '82), pp. 167-169, Paris, France, 1982.

[67] R. R. Yager, "Entropy and specificity in a mathematical theory of evidence," International Journal of General Systems, vol. 9, no. 4, pp. 249-260, 1983.

[68] D. Dubois and H. Prade, "A note on measures of specificity for fuzzy sets," International Journal of General Systems, vol. 10, no. 4, pp. 279-283, 1985.

[69] G. J. Klir and A. Ramer, "Uncertainty in the DempsterShafer theory: a critical re-examination," International Journal of General Systems, vol. 18, no. 2, pp. 155-166, 1990.

[70] G. J. Klir and B. Parviz, "A note on the measure of discord," in Proceedings of the 8th International Conference on Uncertainty in Artificial Intelligence, pp. 138-141, 1992.

[71] T. George and N. R. Pal, "Quantification of conflict in Dempster-Shafer framework: a new approach," International Journal of General Systems, vol. 24, no. 4, pp. 407-423, 1996.

[72] Y. Yang and D. Han, "A new distance-based total uncertainty measure in the theory of belief functions," Knowledge-Based Systems, vol. 94, pp. 114-123, 2016.

[73] Y. Song, X. Wang, L. Lei, and S. Yue, "Uncertainty measure for interval-valued belief structures," Measurement, vol. 80, pp. 241$250,2016$.

[74] Y. Song, X. Wang, and H. Zhang, "A distance measure between intuitionistic fuzzy belief functions," Knowledge-Based Systems, vol. 86, pp. 288-298, 2015.

[75] Y. Deng, "Deng entropy," Chaos, Solitons and Fractals, vol. 91, pp. 549-553, 2016.

[76] R. R. Yager, "On the Dempster-Shafer framework and new combination rules," Information Sciences, vol. 41, no. 2, pp. 93137, 1987.

[77] C. K. Murphy, "Combining belief functions when evidence conflicts," Decision Support Systems, vol. 29, no. 1, pp. 1-9, 2000.

[78] D. Yong, S. WenKang, Z. ZhenFu, and L. Qi, "Combining belief functions based on distance of evidence," Decision Support Systems, vol. 38, no. 3, pp. 489-493, 2004.

[79] Z. Zhang, T. Liu, D. Chen, and W. Zhang, "Novel algorithm for identifying and fusing conflicting data in wireless sensor networks," Sensors, vol. 14, no. 6, pp. 9562-9581, 2014. 
[80] K. Yuan, F. Xiao, L. Fei, B. Kang, and Y. Deng, "Conflict management based on belief function entropy in sensor fusion," SpringerPlus, vol. 5, no. 1, article no. 638, 2016.

[81] W. Jiang, B. Wei, C. Xie, and D. Zhou, "An evidential sensor fusion method in fault diagnosis," Advances in Mechanical Engineering, vol. 8, no. 3, pp. 1-7, 2016. 


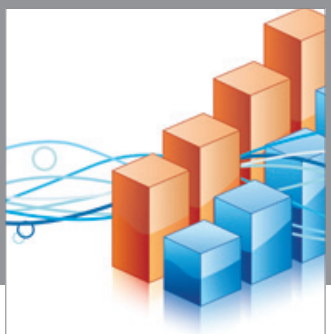

Advances in

Operations Research

vatem alat4

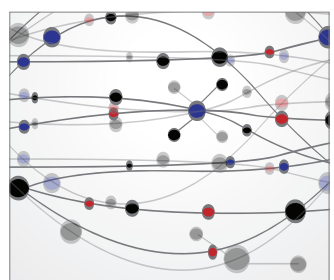

\section{The Scientific} World Journal
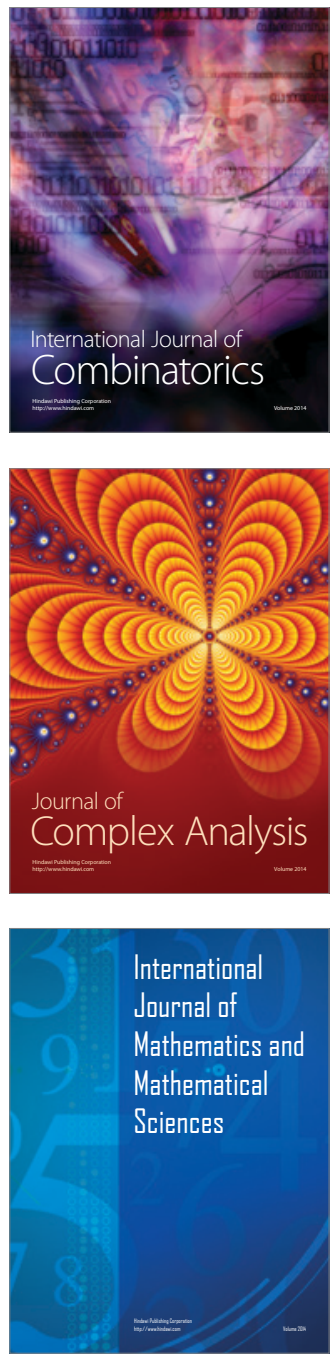
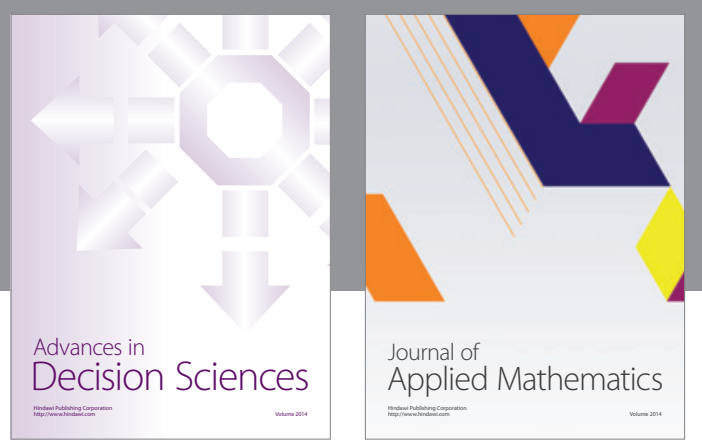

Algebra

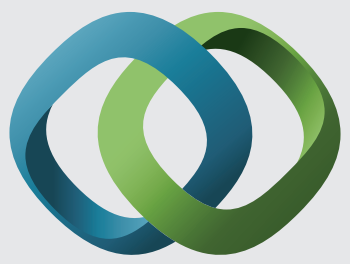

\section{Hindawi}

Submit your manuscripts at

https://www.hindawi.com
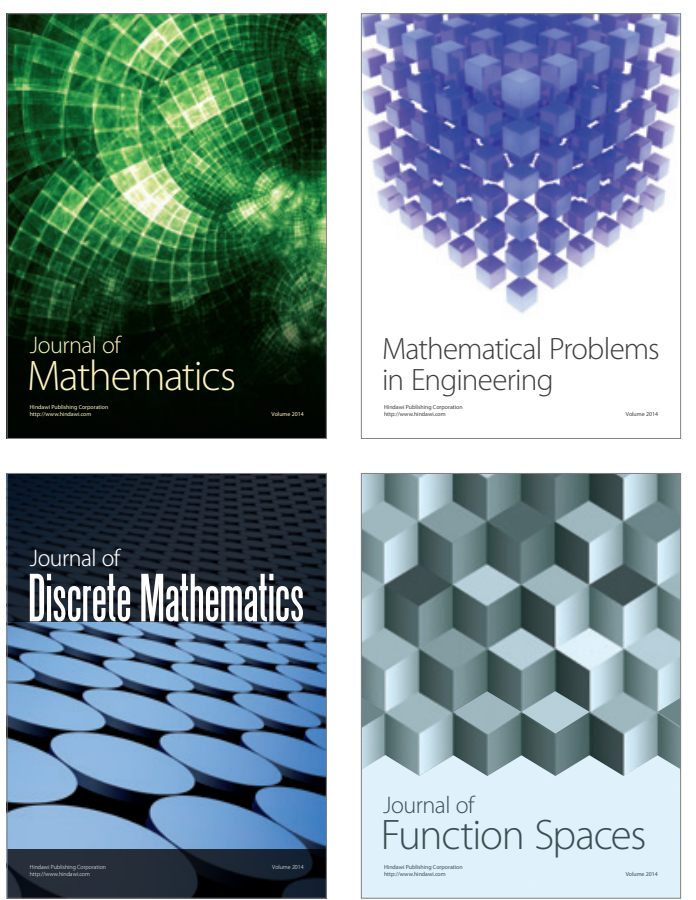

Mathematical Problems in Engineering
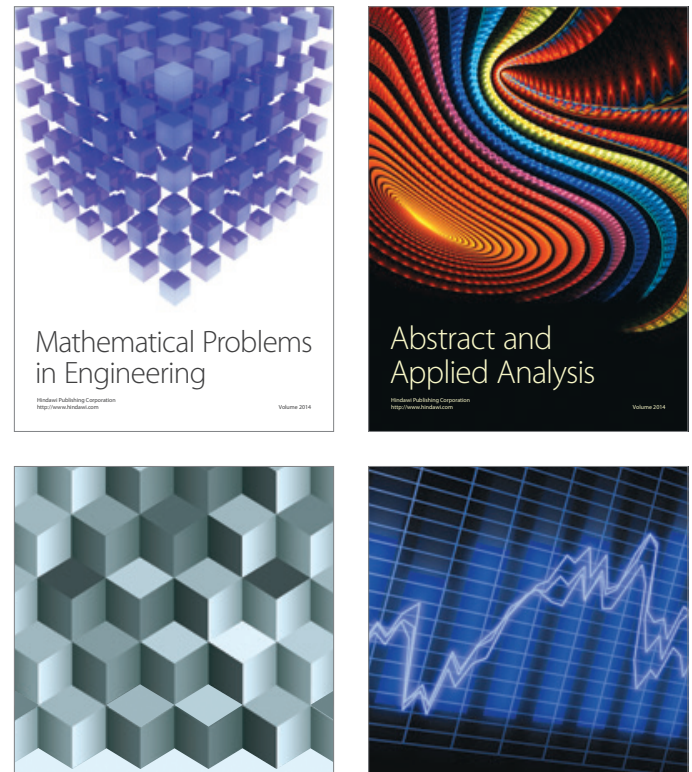

Journal of

Function Spaces

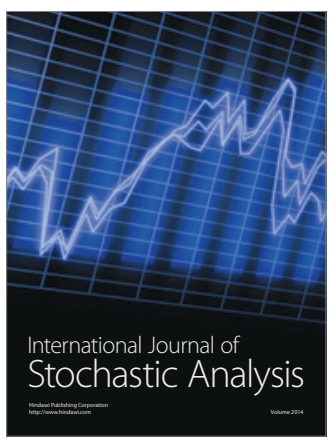

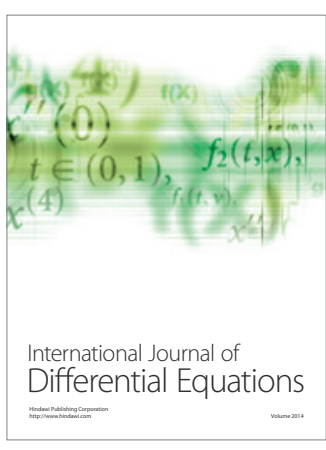
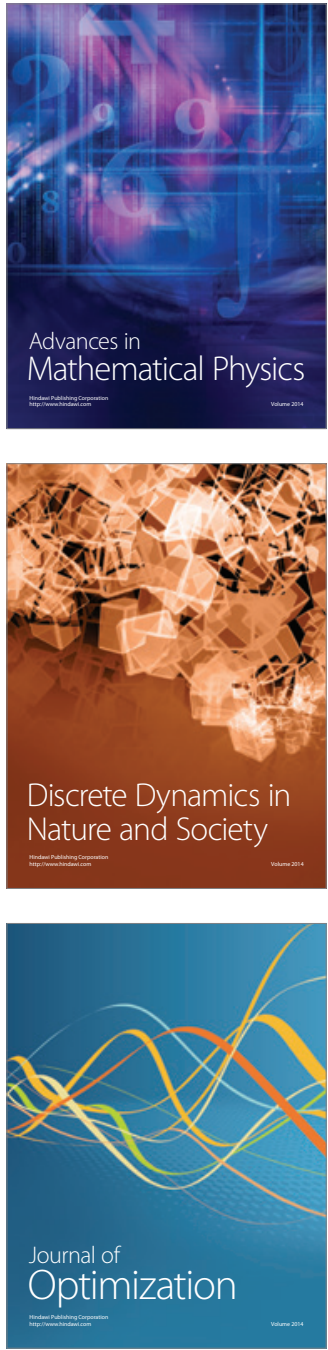